\title{
PROJECTIVELY FLAT AFFINE SURFACES THAT ARE NOT LOCALLY SYMMETRIC
}

\author{
ISAAC CHAUJUN LEE \\ (Communicated by Christopher Croke)
}

\begin{abstract}
By studying affine rotation surfaces (ARS), we prove that any surface affine congruent to $x^{2}+\epsilon y^{2}=z^{r}$ or $y^{2}=z(x+\epsilon z \log z)$ is projectively flat but is neither locally symmetric nor an affine sphere, where $\epsilon$ is 1 or -1 , $r \in \mathbf{R}-\{-1,0,1,2\}$, and $z>0$. The significance of these surfaces is due to the fact that until now $x^{2}+\epsilon y^{2}=z^{-1}$ are the only known surfaces which are projectively flat but not locally symmetric. Although Podestà recently proved the existence of an affine surface satisfying the above italicized conditions, he did not construct any concrete example.
\end{abstract}

\section{Preliminaries}

To study affine differential geometry, we follow the approach of K. Nomizu [N1, N2]. For the affine space $\mathbf{R}^{3}$ with the usual flat connection $D$ and a volume element $\omega$ defined by $\omega\left(\mathscr{Y}_{1}, \mathscr{Y}_{2}, \mathscr{Y}_{3}\right)=\operatorname{det}\left[\mathscr{Y}_{1} \mathscr{Y}_{2} \mathscr{Y}_{3}\right]$, we see that this affine space $\left(\mathbf{R}^{3}, D, \omega\right)$ has an equiaffine structure, that is, $D \omega=0$.

For an affine immersion $f: \mathscr{M}^{2} \longrightarrow \mathbf{R}^{3}$ with a transversal vector field $\eta$, we can define the induced connection $\nabla^{\eta}$, the affine fundamental form $h^{\eta}$, the shape operator $S^{\eta}$, and the transversal connection $\tau^{\eta}$ as follows:

$$
\left\{\begin{aligned}
D_{X} f_{*}(Y) & =f_{*}\left(\nabla^{\eta}{ }_{X} Y\right)+h^{\eta}(X, Y) \eta, \\
D_{X} \eta & =-f_{*}\left(S^{\eta}(X)\right)+\tau^{\eta}(X) \eta .
\end{aligned}\right.
$$

The induced volume element $\theta^{\eta}$ is defined by

$$
\theta^{\eta}\left(Y_{1}, Y_{2}\right)=\omega\left(f_{*}\left(Y_{1}\right), f_{*}\left(Y_{2}\right), \eta\right) .
$$

We have

$$
\left\{\begin{array}{l}
\nabla^{\eta} \text { is a torsion free affine connection, } \\
h^{\eta} \text { is a symmetric tensor, } \\
\nabla_{X} \theta^{\eta}=\tau^{\eta}(X) \theta
\end{array}\right.
$$

We say the affine immersion $f$ is nondegenerate if $h^{\eta}$ is nondegenerate, and we know that this condition is independent of the choice of the transversal vector

Received by the editors January 26, 1993 and, in revised form, April 6, 1993.

1991 Mathematics Subject Classification. Primary 53A15, 53B05, 53C35.

Key words and phrases. Blaschke immersion, locally symmetric, projectively flat, affine rotation surfaces.

This paper is part of a Ph.D. dissertation, Brown University, 1993. 
$\eta$. From now on, we always assume the surface is nondegenerate. We say a transversal vector field $\eta$ is equiaffine ( or $\eta$ is relative normal) if $\nabla^{\eta} \theta^{\eta}=0$.

We have the following fundamental equations for any relative normal:

$\begin{array}{lrl}\text { Gauss Eq. } & R(X, Y) Z & =h(Y, Z) S X-h(X, Z) S Y, \\ \text { Codazzi Eq. } & \left(\nabla_{X} h\right)(Y, Z) & =\left(\nabla_{Y} h\right)(X, Z), \\ \text { Codazzi Eq. } & \left(\nabla_{X} S\right) Y & =\left(\nabla_{Y} S\right) X, \\ \text { Ricci Eq. } & h(S X, Y) & =h(X, S Y) .\end{array}$

The cubic form $C(X, Y, Z):=\nabla_{X} h(Y, Z)$ is symmetric in $X, Y, Z$ by virtue of the Codazzi equation.

Let $Y_{1}, Y_{2}$ be tangent vector fields such that $\theta\left(Y_{1}, Y_{2}\right)=1$ and denote $h^{\eta}\left(Y_{i}, Y_{j}\right)$ by $h_{i j}^{\eta}$. There is an affine invariant, called the affine metric, defined by $h^{\eta} /\left|\operatorname{det}\left(h_{i j}^{\eta}\right)\right|^{1 / 4}$, which is independent of the choice of the transversal vector $\eta$. We then have a unique choice (up to a sign) of a transversal vector field $\xi$, called the affine normal which is equiaffine and satisfies $\theta^{\xi}=\omega_{h}$, where $\omega_{h}$ is the volume element of the affine fundamental form $h^{\xi}$. In this case, $h^{\xi}$ coincides with the affine metric. An affine immersion with affine normal is called a Blaschke immersion. For the shape operator $S$, we define the affine mean curvature $\mathbf{H}$ and the Gauss-Kronecker curvature $\mathbf{K}$ as $\operatorname{trace}(S) / 2$ and $\operatorname{det}(\mathbf{S})$, respectively. A hypersurface is called an affine minimal if the affine mean curvature is zero. A hypersurface is an affine sphere if $S=\lambda \mathbf{I}$, which implies that the function $\lambda$ is actually a constant. If $\lambda \neq 0$, the surface is called a proper affine sphere, and if $\lambda=0$, an improper affine sphere. Let $\hat{\nabla}$ be the corresponding Levi-Civita connection; we can define the scalar curvature as $\widehat{\rho}=\operatorname{trace}_{\mathrm{h}}(\widehat{\gamma}) / 2$, where $\hat{\gamma}$ is the Ricci curvature of the Levi-Civita connection. The Pick invariant is defined by $\mathbf{J}=h(C, C) / 8$. We then have

$$
\widehat{\rho}=\mathbf{H}+\mathbf{J} \text {. }
$$

Two affine connections $\nabla^{1}, \nabla^{2}$ are projectively equivalent if there exists a 1 -form $\rho$ such that

$$
\nabla_{X}^{1} Y-\nabla_{X}^{2} Y=\rho(Y) X+\rho(X) Y .
$$

An affine connection $\nabla$ is called projectively flat if $\nabla$ is projectively equivalent to a flat connection. The following is well known; see, for example, [NP]:

Proposition. An equiaffine immersion $\left(f, \mathscr{M}^{2}, \nabla\right)$ is projectively flat if and only if the Ricci curvature tensor $\gamma$ satisfies the Codazzi equation $\left(\nabla_{X} \gamma\right)(Y, Z)=$ $\left(\nabla_{Y} \gamma\right)(X, Z)$.

Remark. For an affine connection $\nabla$, the definition of the Ricci curvature tensor is $\gamma(Y, Z):=\operatorname{trace}\{X \longrightarrow R(X, Y) Z\}$ where $R$ is the curvature tensor. Note that we can get $\nabla \gamma$ directly from $\nabla R$ as

$$
\nabla_{W} \gamma(Y, Z)=\operatorname{trace}\left\{X \longrightarrow\left(\nabla_{W} R\right)(X, Y) Z\right\}
$$

It is then obvious that the condition $\nabla R=0$ implies projective flatness. The condition $\nabla R=0$ plays an important role in the theory of symmetric spaces. It is known that an affine space $(\mathscr{M}, \nabla)$ is locally symmetric if and only if $\nabla$ is torsion free $(T=0)$ and satisfies $\nabla R=0$ (see [KN, p. 303]).

In $\mathbf{R}^{3}$, we define an affine rotation surface (ARS) as a surface invariant under the action of a 1-parameter subgroup $\{\mathscr{G}(t)\}$ of $\operatorname{AS}(3)$ such that all $\mathscr{G}(t)$ 's have the same line of fixed points. We have 
Theorem [L2]. Any ARS is affinely congruent to one (or more) ${ }^{1}$ of the following standard ARS (SARS): $x^{2}+y^{2}=g_{1}^{2}(z)$ (elliptic type), $x^{2}-y^{2}=g_{2}^{2}(z)$ (hyperbolic type), or $y^{2}=z\left(x-g_{3}(z)\right.$ ) (parabolic type), for some functions $g_{i}(z)$ such that $g_{i}^{\prime \prime}(z) \neq 0$.

We shall study ARS from now on.

\section{MAIN RESULTS}

Theorem 1.1. Any surface affine congruent to one of the following surfaces is projectively flat but not locally symmetric:

(1) $x^{2}+\epsilon y^{2}=z^{r}$ with $r \in \mathbf{R}-\{0,1,2\}, z>0$,

(2) $y^{2}=z(x+\epsilon z \log z)$.

One can see that the first class of surfaces in the theorem contains various classes of surfaces. A further study gives complete information as follows:

Theorem 1.2. Let $\mathscr{M}$ be affine congruent to $x^{2}+\epsilon y^{2}=z^{r}$ for some $r \in$ $\mathbf{R}-\{0,1,2\}, z>0 ;$ then

(1) If $0<r<1$ or $1<r<2$, then, in the case of $\epsilon=1$ (or $\epsilon=-1)$, is definite (respectively, indefinite);

(2) If $r<0$ or $2<r$, then, in the case of $\epsilon=1$ (or $\epsilon=-1$ ), $\mathscr{M}$ is indefinite (respectively, definite).

(3) If $\mathscr{M}$ has non-zero constant Pick invariant, then it is affine congruent to $x^{2}+\epsilon y^{2}=z^{-1}$. In this case, the constant Pick invariant is $\sqrt{2} / \sqrt[4]{2} 7$.

(4) If $\mathscr{M}$ has constant affine Gaussian curvature (or constant affine mean curvature), then it is affine congruent to $x^{2}+\epsilon y^{2}=z^{-1}$. In this case, it is a proper affine sphere.

(5) $\mathscr{M}$ has flat affine metric.

\section{STANDARD AFFINE ROTATION SURFACES (SARS)}

We parametrize the SARS of elliptic, hyperbolic, or parabolic types as follows:

$$
\left\{\begin{array}{l}
{ }^{\mathrm{E}} X(u, v)=g(v){ }^{\mathrm{E}} B(u)+v \quad{ }^{\mathrm{E}} C, \\
{ }^{\mathrm{H}} X(u, v)=g(v){ }^{\mathrm{H}} B(u)+v \quad{ }^{\mathrm{H}} C, \\
{ }^{\mathrm{P}} X(u, v)=v \quad{ }^{\mathrm{P}} B(u)+g(v){ }^{\mathrm{P}} C,
\end{array}\right.
$$

where

$$
\left\{\begin{array}{l}
{ }^{\mathrm{E}} B(u):={ }^{t}(\cos (u), \sin (u), 0), \text { for the elliptic type, } \\
{ }^{\mathrm{H}} B(u):={ }^{t}(\cosh (u), \sinh (u), 0), \text { for the hyperbolic type, } \\
{ }^{\mathrm{P}} B(u):={ }^{t}\left(u^{2} / 2,-u, 1\right), \text { for the parabolic type, } \\
{ }^{\mathrm{E}} C={ }^{\mathrm{H}} C:={ }^{t}(0,0,1),{ }^{\mathrm{P}} C:={ }^{t}(1,0,0) .
\end{array}\right.
$$

And we put $\mathrm{E}$ (or $\mathrm{H}, \mathrm{P}$ ) on the upper-left of an affine quantity if the equation in question is valid for elliptic type (respectively, hyperbolic type, parabolic type),

\footnotetext{
${ }^{1}$ For example, the hyperboloid of one sheet $x^{2}+y^{2}-z^{2}=1$ can be of all three types (see [L2, p. 49]).
} 
and omit these upper-left indicies if the equation is valid for all types. We also denote $X_{, 1}:=\frac{\partial X}{\partial u}, X_{, 2}:=\frac{\partial X}{\partial v}, B^{\prime}:=\frac{\partial B}{\partial u}$, and

$$
\left\{\begin{array}{l}
\phi:=\left(\left|\delta^{3} g^{\prime \prime}\right|\right)^{\frac{1}{4}}, \quad \tau_{2}:=-(\delta \mu)^{\prime} /(\delta \mu), \\
T_{2}:=\left(-\phi \tau_{2}-\phi^{\prime}\right) /\left(\delta g^{\prime \prime}\right)=-{ }^{\mathrm{P}} T_{2},
\end{array}\right.
$$

where

$$
(\delta, \mu, \psi):= \begin{cases}\left(g, g^{\prime}, 1\right) & \text { for elliptic/hyperbolic ARS }, \\ \left(v, 1, g^{\prime}\right) & \text { for parabolic ARS. }\end{cases}
$$

Regarding $\left\{X_{, 1}, X_{, 2}, \xi\right\}$ and $\left\{B, B^{\prime}, C\right\}$ as two bases of $\mathbf{R}^{3}$, we have

$$
(X, 1, X, 2, \xi)=\left(B, B^{\prime}, C\right) \cdot \mathscr{P} \quad \text { with } \mathscr{P}=\left(\begin{array}{ccc}
0 & \mu & T_{2} \mu \\
\delta & 0 & 0 \\
0 & \psi & T_{2} \psi-\phi /(\mu \delta)
\end{array}\right) .
$$

Differentiating this with respect to $u$ and $v$, we have

$$
\left\{\begin{array}{l}
\left(X_{, 11}, X_{, 21}, \xi_{, 1}\right)=\left(X_{, 1}, X_{, 2}, \xi\right) \cdot \mathscr{P}-1 \cdot \mathscr{L} \cdot \mathscr{P}, \\
\left(X_{, 12}, X_{, 22}, \xi_{, 2}\right)=\left(X_{, 1}, X_{, 2}, \xi\right) \cdot \mathscr{P}-1 \cdot \mathscr{P} \prime
\end{array}\right.
$$

where

$$
\mathrm{E} \mathscr{L}=\left(\begin{array}{ccc}
0 & -1 & 0 \\
1 & 0 & 0 \\
0 & 0 & 0
\end{array}\right), \quad \mathrm{H} \mathscr{L}=\left(\begin{array}{lll}
0 & 1 & 0 \\
1 & 0 & 0 \\
0 & 0 & 0
\end{array}\right), \quad \text { and } \quad \mathrm{P} \mathscr{L}=\left(\begin{array}{lll}
0 & 0 & 0 \\
1 & 0 & 0 \\
0 & 1 & 0
\end{array}\right) \text {. }
$$

Therefore, we obtain

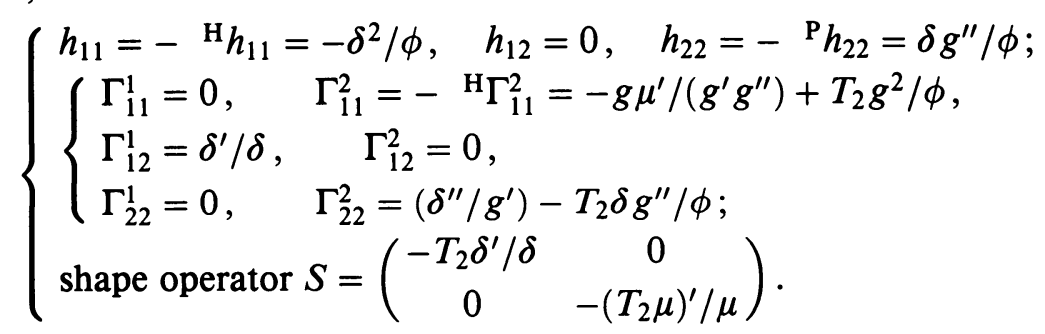

Furthermore, we can eliminate $T$ and $\mu$ in $\Gamma_{11}^{2}, \Gamma_{22}^{2}$ by using (2.1) and (2.2). We get

$$
\Gamma_{11}^{2}=\left(-\frac{1}{4}\right)\left(\delta / g^{\prime \prime}\right)^{\prime}=-{ }^{\mathrm{E}} \Gamma_{11}^{2}, \quad \Gamma_{22}^{2}=\left(-\frac{1}{4}\right)\left(\delta / g^{\prime \prime}\right)^{\prime} /\left(\delta / g^{\prime \prime}\right) .
$$

Note that $\Gamma_{22}^{1}=0$ implies that any $v$-curve is a pregeodesic. We say a SARS has geodesic parameters if $\Gamma_{11}^{2}=0$.

It is known that SARS of elliptic type share many affine properties with those of hyperbolic type in the following sense:

Proposition 2.1 [L2, p. 38]. For any SARS of elliptic type and that of hyperbolic type with the same function $g(v)$, each of the following properties holds for one surface if and only if it does for the other.

(1) An orbit passing through a point $p_{0}$ of the profile curve is a geodesic relative to the induced connection.

(2) An orbit passing through a point $p_{0}$ of the profile curve is a geodesic relative to the Levi-Civita connection of the affine metric. 
(3) An orbit passing through a point $p_{0}$ of the profile curve is a shadow boundary.

(4) The surface is a quadric.

(5) The surface has constant Pick invariant.

(6) The surface is a proper affine sphere.

(7) The surface is an improper affine sphere (or equivalently, flat).

(8) The surface has constant mean curvature.

(9) The surface has constant Gauss-Kronecker curvature.

(10) The surface is locally symmetric.

(11) The surface is projectively flat.

(12) The affine metric has constant curvature.

Because of this proposition, we usually study SARS of elliptic type.

\section{TWO LEMMAS}

We shall simplify the expression of $\nabla R$ by writing $\left(\nabla_{m} R_{j k l}^{i}\right) \frac{\partial}{\partial x_{i}}$ or $R_{j k l ; m}^{i} \frac{\partial}{\partial x_{i}}$ for $\left(\nabla_{\frac{\partial}{\partial x_{m}}} R\right)\left(\frac{\partial}{\partial x_{k}}, \frac{\partial}{\partial x_{l}}, \frac{\partial}{\partial x_{j}}\right)$. We compute $\nabla R$ for SARS by using (2.4) and have

$$
\left\{\begin{array}{l}
\nabla_{1} R_{112}^{1}=-\nabla_{1} R_{212}^{2}=-\nabla_{1} R_{121}^{1}=\nabla_{1} R_{221}^{2}=R_{112}^{2} \Gamma_{21}^{1}-R_{212}^{1} \Gamma_{11}^{2}, \\
\nabla_{2} R_{112}^{2}=-\nabla_{2} R_{121}^{2}=\left(R_{112}^{2}\right)^{\prime}-2 R_{112}^{2} \Gamma_{12}^{1}, \\
\nabla_{2} R_{212}^{1}=-\nabla_{2} R_{221}^{1}=\left(R_{212}^{1}\right)^{\prime}-2 R_{212}^{1} \Gamma_{22}^{2} \\
\nabla_{l} R_{k i j}^{t}=0, \quad \text { otherwise, }
\end{array}\right.
$$

where

$$
\left\{\begin{array}{l}
R_{112}^{2}=-\left(\Gamma_{11}^{2}\right)^{\prime}+\Gamma_{11}^{2} \Gamma_{21}^{1}-\Gamma_{11}^{2} \Gamma_{22}^{2} \\
R_{212}^{1}=-\left(\Gamma_{21}^{1}\right)^{\prime}-\Gamma_{21}^{1} \Gamma_{21}^{1}+\Gamma_{21}^{1} \Gamma_{22}^{2}
\end{array}\right.
$$

Then we have

Lemma 3.1. Any SARS with geodesic parameters is locally symmetric.

Lemma 3.2. An SARS is projectively flat if and only if its Christoffel symbols satisfy

$$
\left(\left(\Gamma_{11}^{2}\right)^{\prime}-2 \Gamma_{11}^{2} \Gamma_{12}^{1}+\Gamma_{11}^{2} \Gamma_{22}^{2}\right)^{\prime}=0 .
$$

Proof of Lemma 3.1. We know that an SARS has geodesic parameters if and only if $\Gamma_{11}^{2}=0$. We now prove that $\Gamma_{11}^{2}=0$ implies local symmetry, namely, $\nabla R \equiv 0$.

If $\Gamma_{11}^{2}=0,(3.2)$ becomes

$$
\left\{\begin{array}{l}
R_{112}^{2}=0, \\
R_{212}^{1}=\left(-\left(\Gamma_{21}^{1}\right)^{\prime}-\Gamma_{21}^{1} \Gamma_{21}^{1}\right)+\Gamma_{21}^{1} \Gamma_{22}^{2} .
\end{array}\right.
$$

Plugging these into (3.1), we have

$$
\begin{gathered}
\nabla_{1} R_{121}^{1}=0, \\
\nabla_{2} R_{121}^{2}=0, \\
\nabla_{2} R_{212}^{1}=-\nabla_{2} R_{221}^{1}=\left(R_{212}^{1}\right)^{\prime}-2 R_{212}^{1} \Gamma_{22}^{2} .
\end{gathered}
$$

We now prove the last equation is zero by showing $\left(R_{212}^{1}\right)^{\prime}=0$ and $\Gamma_{22}^{2}=0$ as follows. 
We express $\Gamma_{12}^{1}$ and $\Gamma_{22}^{2}$ in terms of $\Gamma_{11}^{2}$.

(I) From (2.4) and (2.5), we have

$$
\left\{\begin{array}{l}
\left(\frac{1}{\left(\Gamma_{21}^{1}\right)^{\prime}+\Gamma_{21}^{1} \Gamma_{21}^{1}}\right)^{\prime}=-4 \Gamma_{11}^{2} \text { for parabolic type } \\
\left(\Gamma_{21}^{1}\right)^{\prime}+\Gamma_{21}^{1} \Gamma_{21}^{1}=0 \text { for elliptic/hyperbolic type. }
\end{array}\right.
$$

(II) From (2.5), we have

$$
\Gamma_{22}^{2}=\Gamma_{11}^{2} /\left(-4 \int \Gamma_{11}^{2} \mathrm{~d} v\right) \text {. }
$$

Now, with the assumption $\Gamma_{11}^{2}=0$, we have $\Gamma_{22}^{2}=0$ by (3.5). Thus, (3.1) becomes

$$
\left\{\begin{array}{l}
\nabla_{2} R_{212}^{1}=-\nabla_{2} R_{221}^{1}=\left(R_{212}^{1}\right)^{\prime}, \\
\nabla_{l} R_{k i j}^{t}=0, \quad \text { otherwise. }
\end{array}\right.
$$

Furthermore, we can use (3.4) and (3.2) to prove $\left(R_{212}^{1}\right)^{\prime}=0$. Therefore, $\nabla R \equiv 0$.

Remark. Any ARS with geodesic parameters (relative to the affine normal) is affine congruent to one of the following classes [L2, p. 66]:

$$
\begin{array}{cc}
x^{2}+\epsilon y^{2}=\cos ^{2} z, & x^{2}+\epsilon y^{2}=\sinh ^{2} z, \\
x^{2}+\epsilon y^{2}=\cosh ^{2} z, & y^{2}=x z+\epsilon z^{4} .
\end{array}
$$

Proof of Lemma 3.2. We only have to check equations $\gamma_{j[k ; i]}=0$, which are basically two equations $\gamma_{1[1 ; 2]}=0$ and $\gamma_{2[1 ; 2]}=0$. By $(0.3)$, we have

$$
\gamma_{2[1 ; 2]}=\gamma_{21 ; 2}-\gamma_{22 ; 1}=R_{1 m 2 ; 2}^{m}-R_{2 m 2 ; 1}^{m}=R_{112 ; 2}^{2}-R_{212 ; 1}^{2} \underset{(3.1)}{=} 0 .
$$

Similarily, we have $\gamma_{1[1 ; 2]}=\left(R_{121}^{2}\right)^{\prime}-R_{121}^{2} \Gamma_{21}^{1}-R_{221}^{1} \Gamma_{11}^{2}$. Using (3.2) and (2.4), we can express $\gamma_{1[1 ; 2]}$ in terms of the Christoffel symbols of SARS as $\left(\left(\Gamma_{11}^{2}\right)^{\prime}-2 \Gamma_{11}^{2} \Gamma_{12}^{1}+\Gamma_{11}^{2} \Gamma_{22}^{2}\right)^{\prime}$.

\section{Proof of Theorem 1.1}

For each type of SARS, the differential equation (3.3) in Lemma 3.2 can be expressed as a 5th order nonlinear ordinary differential equation of $g(v)$ by using the notions in (2.4) and (2.1). If we want to look for SARS that are projectively flat, we have to solve these 5 th order ordinary differential equations, whose general solutions seem to be difficult to find. Theorem 1.1 is obtained by assuming each term of (3.3) is zero. In orther words, we want to solve the following simultaneous differential equations for arbitary constants $a, b, c$ :

$$
\left\{\begin{array}{l}
\text { (i) }\left(\Gamma_{11}^{2}\right)^{\prime}=a, \\
\text { (ii) } \Gamma_{11}^{2} \Gamma_{12}^{1}=b, \\
\text { (iii) } \Gamma_{11}^{2} \Gamma_{22}^{2}=c .
\end{array}\right.
$$

These constants are in fact subject to (4.2) as follows:

Condition (i) implies that $\Gamma_{11}^{2}=a v+d$ for some constant $d$ and then $\frac{1}{4} \delta / g^{\prime \prime}=\int \Gamma_{11}^{2} \mathrm{~d} v=a v^{2} / 2+d v+e$ for some constant $e$. We use (3.5) to get 
constraints on $a, d, e, c$ from conditions (i) and (iii):

$$
\left\{\begin{array}{l}
c=-a / 2, \\
2 a e=d^{2} .
\end{array}\right.
$$

If $a=0$, then $c=0, d=0$. Thus, $\Gamma_{11}^{2}=a v+d=0$, which corresponds to locally symmetric surfaces by virtue of Lemma 3.1 .

From now on, we assume $a \neq 0$. Then, (4.2) becomes

$$
\left\{\begin{array}{l}
c=-a / 2 \neq 0 \\
e=d^{2} /(2 a)
\end{array}\right.
$$

namely,

$$
\int \Gamma_{11}^{2} \mathrm{~d} v=(a / 2)(v+d / a)^{2} .
$$

Remark. If $\Gamma_{11}^{2}=a v$, then $\int \Gamma_{11}^{2} \mathrm{~d} v=a v^{2} / 2$.

We now proceed to discuss the solution for the case of elliptic/hyperbolic type and that of parabolic type.

(1) For elliptic/hyperbolic type. We will find $g(v)$ for projectively flat SARS of elliptic typs; then the same $g(v)$ works for that of hyperbolic type by virtue of Proposition 2.1.

From condition (i) of (4.1), we can assume

$$
\Gamma_{11}^{2}=a v+d
$$

Then, condition (ii) implies

$$
\Gamma_{12}^{1}=g^{\prime} / g=\frac{b}{(a v+d)},
$$

which has solution

$$
g(v)=n(a v+d)^{\frac{b}{a}} .
$$

Note that $a \neq b$, otherwise $g^{\prime \prime}(v)=0$ corresponding to a degenerate surface.

Claim. $g(v)=n(a v+d)^{1+1 / 2 b}$. Since

$$
\begin{aligned}
a v+d & =\Gamma_{11}^{2} \quad(\text { by }(4.5)) \\
& =(1 / 4)\left(g / g^{\prime \prime}\right)^{\prime} \quad(\text { by }(2.4)) \\
& =a(a v+d) /(2 b(b-a)) \quad(\text { by }(4.7)),
\end{aligned}
$$

we have a constraint $a=2 b^{2} /(1+2 b), b \in \mathbf{R}-\{0,-1 / 2\}$. Plugging this into (4.7) proves our claim.

Let

$$
1+1 / 2 b=r / 2, \quad r \in \mathbf{R}-\{0,2\} .
$$

Thus, for any $r \in \mathbf{R}-\{0,2\}$, the surface $x^{2}+\epsilon y^{2}=z^{r}$ is projectively flat.

To prove those surface with $r \in \mathbf{R}-\{0,1,2\}$ is not locally symmetric, we rewrite $\nabla_{2} R_{112}^{2}$ in terms of $r$ as follows:

$$
\begin{aligned}
\nabla_{2} R_{112}^{2} & =\left(R_{112}^{2}\right)^{\prime}-2 R_{112}^{2} \Gamma_{12}^{1} \quad(\text { by }(3.1)) \\
& =(-a+b-c)^{\prime}-2(-a+b-c) \Gamma_{12}^{1} \quad(\text { by }(3.2) \text { and }(4.1)) \\
& =-(r-1) /(v(r-2)) \quad(\text { by }(4.3),(4.6), \quad \text { and }(4.8)),
\end{aligned}
$$

which is not 0 since $r \in \Lambda:=\mathbf{R}-\{0,1,2\}$.

These prove our assertion for surfaces in class (1). 
(2) For parabolic type. From condition (i) of (4.1), we can assume $\Gamma_{11}^{2}=$ $a v+d$. Together with $\Gamma_{12}^{1}=1 / v$ from (2.4), condition (ii) implies

$$
a=b \quad \text { and } \quad d=0 .
$$

On the other hand, since $\Gamma_{11}^{2}=\frac{-1}{4}\left(v / g^{\prime \prime}\right)^{\prime}$, we have

$$
\frac{1}{4}\left(v / g^{\prime \prime}\right)=\int \Gamma_{11}^{2} \mathrm{~d} v=\frac{a v^{2}}{2} \quad(\text { by (4.4) and (4.9)). }
$$

Solving this equation, we have $g(v)=v \log (v) /(-2 a)+l v+m$. It gives a surface affinely congruent to $y^{2}=z(x+\epsilon z \log z)$.

To prove that these are not locally symmetric, we rewrite $\nabla_{2} R_{112}^{2}$ in terms of $a$ as follows:

$$
\begin{aligned}
\nabla_{2} R_{112}^{2} & =\left(R_{112}^{2}\right)^{\prime}-2 R_{112}^{2} \Gamma_{12}^{1} \quad(\text { by }(3.1)) \\
& =(-a+b-c)^{\prime}-2(-a+b-c) \Gamma_{12}^{1} \quad(\text { by }(3.2) \text { and }(4.1)) \\
& =-a / v \quad(\text { by }(4.3) \text { and }(4.9)),
\end{aligned}
$$

which is not 0 since $a \neq 0$. Therefore, these surfaces are not locally symmetric.

The assertion for the surfaces in class (2) is proven.

\section{Proof of TheORem 1.2}

For statements (1) \& (2). An affine surface is definite (indefinite) if and only if a matrix representation $\left[h_{i j}\right]$ of its second fundamental form satisfies $\operatorname{det}\left[h_{i j}\right]>0$ (respectively, $\left.\operatorname{det}\left[h_{i j}\right]<0\right)$. By $(2.4)$, for $x^{2}+\epsilon y^{2}=g^{2}(z)$, we have

$$
\left[{ }^{\mathrm{E}} h_{i j}\right]=\left(\begin{array}{cc}
-g^{2} / \phi & 0 \\
0 & g g^{\prime \prime} / \phi
\end{array}\right) \text { and }\left[{ }^{\mathrm{H}} h_{i j}\right]=\left(\begin{array}{cc}
g^{2} / \phi & 0 \\
0 & g g^{\prime \prime} / \phi
\end{array}\right) \text {. }
$$

Thus, $\operatorname{det}\left[{ }^{\mathrm{E}} h_{i j}\right]=-g^{3} g^{\prime \prime} /\left(\phi^{2}\right)=-\operatorname{det}\left[{ }^{\mathrm{H}} h_{i j}\right]$. For the case of $x^{2}+\epsilon y^{2}=z^{r}$, we have $g(v)=v^{(r / 2)}$. Computation shows

$$
\operatorname{det}\left[{ }^{\mathrm{E}} h_{i j}\right]=\frac{-r(r-2) v^{2 r-2}}{4 \phi^{2}}=-\operatorname{det}\left[{ }^{\mathrm{H}} h_{i j}\right] .
$$

Determining the region of $r$ such that $-r(r-2)>0$ or $-r(r-2)<0$, statements (1) and (2) follow.

For statement (3). By virtue of statement (5) of Proposition 2.1, we only have to prove the case of elliptic type $x^{2}+y^{2}=z^{r}$.

We use (2.4) to compute the Pick invariant $\mathbf{J}$ and have $\mathbf{J}=\left(\frac{1}{2}\right)\left(C_{112} h^{11}\right)^{2} h^{22}$, where $C_{112}=h_{12,1}-\Gamma_{11}^{2} h_{22}-\Gamma_{12}^{1} h_{11}$.

By using (5.1), (2.4), (2.5) and knowing $g=v^{(r / 2)}$, we have

$$
\begin{gathered}
\phi=\sqrt[4]{r(r-2) v^{2 r-2} / 4}, \\
h_{11}=-v^{r} / \phi, \quad h_{22}=(r)(r-2) v^{r-2} /(4 \phi), \\
\Gamma_{11}^{2}=2 v / r(r-2), \quad \Gamma_{12}^{1}=r / 2 v, \quad \Gamma_{22}^{2}=-1 / 2 v .
\end{gathered}
$$

Thus, we have $(-r) \Gamma_{11}^{2} h_{22}=\Gamma_{12}^{1} h_{11}$ and then obtain

$$
\mathbf{J}=\frac{(r-1)^{2} \phi}{\left(2 r(r-2) v^{r}\right.}=\frac{(r-1)^{2}}{2 \sqrt[4]{4 r^{3}(r-2)^{3} v^{2 r+2}}} .
$$

Therefore, $\mathbf{J}$ is a constant if and only if the exponent of $v$ is zero, i.e., $r=-1$. In this cases, $\mathbf{J}=\sqrt{2} / \sqrt[4]{2} 7$. 
For statement (4). To compute the shape operator, we use (2.4) and have $\tau_{2}=-(r-1) / v$, and then

$$
T_{2}=\frac{2 \phi(r-1)}{r(r-2) v^{r-1}} .
$$

Thus, by (2.4), we can show

$$
\left\{\begin{array}{l}
S_{11}=-r(r-1) \phi /\left(r(r-2) v^{r}\right)=-r(r-1) /\left(\sqrt[4]{4 r^{3}(r-2)^{3} v^{2 r+2}}\right) \\
S_{22}=(r-1) \phi /\left(r(r-2) v^{r}\right)
\end{array}\right.
$$

Note that $S_{11}=(-r) S_{22}$.

Therefore, the surface has constant affine Gaussian curvature (or constant affine mean curvature) if and only if the exponent of $v$ of $S_{11}$ is zero, i.e., $r=-1$. In this case, $S_{11}=S_{22}=-\sqrt{2} / \sqrt[4]{2} 7$.

For statement (5). To see that the curvature $\widehat{\kappa}$ of the affine metric is zero, we simply refer to (5.3) and have

$$
\mathbf{H}=\left(S_{11}+S_{22}\right) / 2=\left(\frac{-r+1}{2}\right) S_{22}=-\frac{\left(r^{2}-1\right) \phi}{2 r(r-2) v^{r}} .
$$

Comparing this with (5.2), we conclude that $\mathbf{H}=-\mathbf{J}$. Therefore, we have $\widehat{\kappa}=\widehat{\rho}=0$ by $(0.2)$.

Remark. As an analogue of statements (4) and (5) of Theorem 1.2, one can show that any projectively flat surface $y^{2}=z(x+\epsilon z \log z)$ has flat affine metric but is not an affine sphere. These surfaces suggest the classification of projectively flat affine surfaces with flat affine metric. Note that the classification will be complete once one classifies those surfaces that are not affine spheres. The reason is that affine spheres are projectively flat and that affine spheres with flat affine metric are classified by M. Magid and P. Ryan [MR]. Furthermore, U. Simon [S] classified affine spheres with constant curvature metric. Thus, a natural generalization is to classify projectively flat affine surfaces with constant curvature metric. In the case of affine rotation surfaces, we can prove that any ARS that is an affine sphere with constant curvature metric is a quadric or affine congruent to $x^{2}+\epsilon y^{2}=1 / z$.

\section{ACKNOWLEDGMENT}

The author would like to thank Professor K. Nomizu for his guidance and contiunous encouragement.

\section{REFERENCES}

[KN] S. Kobayashi and K. Nomizu, Foundations of differential geometry, Vol. I, Wiley, New York, 1969.

[L1] C. Lee, Affine rotation surfaces, Master's Thesis, Brown Univ., 1991.

[L2] - Generalized affine rotation surfaces, Ph.D. Thesis, Brown Univ., 1993.

[MR] M. Magid and P. Ryan, Flat affine spheres in $\mathbf{R}^{3}$, Geom. Dedicata 33 (1990), 277-288.

[N1] K. Nomizu, What is affine differential geometry, Proc. Conference on Diff. Geom., Munster, 1982, pp. 42-43.

[N2] _ Introduction to affine differential geometry, Part I, Lecture Notes, MPI preprint MPI 88-37, 1988; revised: Department of Mathematics, Brown University, 1989. 
[NP] K. Nomizu and U. Pinkall, On a certain class of homogeneous flat manifolds, Tôhoku Math. J. 39 (1987), 407-427.

[Po] F. Podestà, Projectively flat surfaces in $\mathrm{A}^{3}$, Proc. Amer. Math. Soc. 119 (1993), 255-260.

[S] U. Simon, Local classification of two-dimensional affine spheres with constant curvature metric, Differential Geometry Appl. 1 (1991), 123-132.

Department of Mathematics, Brown University, Providence, Rhode Island 02912

Current address: Department of Applied Mathematics, National Sun Yet-Sen University, Kaohsiung, Taiwan 80424

E-mail address: isaac@ibm23.math.nsysu.edu.tw 